\title{
La autobiografía del P. Diego de Boroa
}

The autobiography of Father Diego de Boroa

Silvana M. Lovay ${ }^{*}$

Resumen: Los jesuitas impulsaron el género biográfico con dos fines particularmente precisos, uno motivado por las continuas críticas a la Compañía de Jesús y otro muy importante, el de dar a conocer y poner en relevancia a sus miembros como modelos de santidad, de virtud y de piedad, conformando de este modo un rico acervo documental. En este caso trabajamos una autobiografía, como documento poco usual, particularmente en la provincia del Paraguay. Nos referimos a un relato de su propia vida, escrita por el P. Diego de Boroa, quien hace un detallado recorrido de su andar, desde la infancia hasta su ingreso a la Orden, donde podremos no solo conocer sobre su opción cristiana, sino poner en valor su devoción y su dedicación manifiesta en el otro, descubriendo de este modo su real vocación por los excluidos, mundo en el que se siente uno más entre de ellos.

Palabras clave: Diego de Boroa, Jesuitas del Paraguay, biografías

\begin{abstract}
The Jesuits drove the biographical genre with two particularly specific purposes, one motivated by the continuous criticism of the Order and other very importantly, to publicize and implement relevant members of the Company as models of holiness, virtue and piety, thereby forming a rich documentary heritage. In this case we work one autobiography, as unusual document, particularly in the province of Paraguay. We refer to an account of his life written by Father Diego de Boroa, who makes a detailed tour of his walk, from childhood to his admission to the Society of Jesus, where we can not only learn about their Christian option but to value his devotion and dedication put into the other, thus discovering his true vocation for the excluded, the world in which one feels more of them.
\end{abstract}

Key words: Diego de Boroa, Jesuits of Paraguay, biographies

Recibido: 16 de marzo de 2015

Evaluado: 28 de mayo de 2015

* Licenciada en Gestión de Instituciones Educativas, Magister en Museología y Doctoranda en Ciencias de la Educación. Investigadora del programa Jesuitas en Iberoamérica del CIECS-CONICET/UNC. Email: lovaysilvi@hotmail.com. Argentina. 
Los jesuitas promovieron el género biográfico de manera importante, dentro de sus textos históricos, y hasta fue el inicio de importantes proyectos historiográficos. Esta vocación se alentó por varios motivos, pero al menos hasta el primer centenario (1640) lo hicieron ante las continuas y muy duras críticas hacia la Compañía de Jesús que soportaron desde su fundación. Una de ellas y la más sensible fue la de Hieronymo Zahorowski que, en su conocida obra publicada en 1624, denunciaba que los jesuitas querían acaparar dinero y poder para apoderarse del mundo. Una manera de contrarrestarlas fue redactar su propia historia, pero tenían la desventaja de ser una Orden nueva, con escaso pasado frente a las otras; por tanto trabajaron en resaltar las cualidades de sus miembros como modelos de santidad, de virtud y de piedad. En este sentido los mártires de las misiones americanas y orientales fueron emblemáticas, ensalzando virtudes un tanto narcisistas como afirma García Cárcel ${ }^{1}$ y de evidente tono apologético. Aunque tampoco fue la única Orden que usó este género historiográfico, pero no se puede negar que era una buena estrategia, donde la biografía se constituyó en el eje de su historia.

Las Cartas de Edificación eran los primeros escritos que redactaban los jesuitas, generalmente testigos o vinculados al fallecido. Inmediatamente después eran enviadas al provincial quien las resumía o transcribía textualmente a los fines de incluirla en las Cartas Anuas como necrológicas u obituarios. Por otra parte, los Elogios eran las biografías con una fecha que se destacaba en la vida del biografiado y servía, tanto para los menologios como para la recopilación de "Vidas Ejemplares". En este sentido el jesuita toledano Pedro de Ribadeneyra comenzó la tarea en 1594, escribiendo sobre los tres primeros generales. Con este material el general Aquaviva, le encargó al P. Nicolò Orlandi la redacción de una historia con base biográfica y con una metodología que se emplearía siempre (incluso en el siglo XIX): enviar una serie de preguntas formales a las diferentes provincias, para que sean respondidas a través de los testimonios de los más ancianos y sus propios archivos; tarea en la que quedaría a cargo el que se llamó "historiador de provincia". Fue entonces que nació este oficio dentro de la Compañía de Jesús. Entre las cuestiones solicitadas por el P. Aquaviva destaquemos que debían remitir: "Algunas virtudes y acciones especiales de aquellos que murieron dentro de la Compañía: santidad de vida, muerte preclara, enlistados los nombres y demás circunstancias"2.

Comenzaron a llegar a Roma centenares de manuscritos, cuyos destinos en el tiempo fueron variados, pero en general, si bien es muy rico el acervo documental jesuita existente y disperso por el mundo, la mayoría de esos documentos desparecieron.

Es de destacar que el P. Alfonso Polanco organizó el archivo, presidiendo la labor del P. Orlandi, quien no pudo concluir su extensa tarea, y le sucedieron Francesco Sacchini, el francés Joseph de Jouvancy y Giulio Cordara.

De nuestra región fue la época en que se publicaron las conocidas obras de los PP. Ovalle, Vasconcelos, Ruiz de Montoya y Del Techo, no corriendo igual suerte el manuscrito perdido del P. Juan Pastor (1580-1658), considerado el primer historiador de la provincia paraguaya ${ }^{3}$.

\footnotetext{
${ }^{1}$ García Cárcel, 2010: 55.

2 Alcántara Bojorge, 2009: 69.

${ }^{3}$ Su obra "Historia de la Compañía de Jesús en la Provincia del Paraguay" la concluyó en 1649. Posteriormente y con el apoyo de los censores de la provincia la presentó al P. General Goswino Nickel para su aprobación. Pero no se le permitió imprimirla al argumentarse en 1654 que si bien "todos dicen mucho
} 
Después de esta experiencia vendría un proyecto historiográfico más complejo, motivado por la celebración del primer centenario de la Orden (1640), cuyo producto más representativo fue la obra sobre la provincia Frandro-Belga, aparecida en ese año y dirigida por el P. Bolland, el gran historiador que dio un giro importante a la manera de reconstruir el pasado. Pero las biografías continuaron siendo el eje de esas historias, aunque ahora se sumaban otros individuos a las vidas de los generales.

En nuestro caso, las Decades del mencionado P. Nicolás Del Techo (16111685), es la obra más conocida de su tiempo, publicada recién en 1759 por el P. Orosz, muchos años después de la muerte del historiador en 1685, aunque con nula difusión. Igualmente la obra de Del Techo circulaba entre los jesuitas. Efectivamente un manuscrito que se halla en la Biblioteca Nacional de España, delicadamente escrito, contiene como encabezado: "Este tomo esta escrito por los mismos Indios del Paraguay de la antigüedad, imitando la letra de imprenta”, y un exlibris: "Dela librería del Col. ${ }^{\circ}$ de Jhs de Cordova”. Está dedicado al general Carlos de Noyelle (1682-1686) y estampa su firma manuscrita el mismo P. Nicolás Del Techo. Contiene las biografías de 39 jesuitas que son las que toma después el P. Orosz.

El proyecto historiográfico del Centenario vio sus saludables frutos en la primera mitad del siglo XVIII. Después comenzó a disminuir la producción debido a las sucesivas expulsiones. Justamente recién, se reiniciará en el periodo siguiente a la Restauración, con el general P. Luis Martín, a fines del siglo XIX.

\section{El P. Diego de Boroa}

Llegado en la primera expedición al Paraguay de 1610, conducida por el procurador P. Juan Romero, el P. Boroa (Trujillo, 1585 - San Miguel, 1657) fue partícipe importante en la construcción de la historia misional y estaba totalmente imbuido en la metodología del género biográfico. De hecho era uno de los tantos jesuitas que enviaban sus contribuciones a Roma, sin el ánimo que sean publicadas sino fundamentalmente a los fines que sean utilizadas por los historiadores de la Orden. Como hizo y reconoce el P. Rivanedeyra, que escribió sobre el P. Lorenzana en base a la obra del P. Boroa, aunque desconocemos si este último solicitó licencia para publicarla. Sí sabemos que la utilizó tanto Del Techo como Lozano, quienes basaron sus historias en la obra del P. Boroa y, por cierto, en la desparecida del P. Pastor que citan con frecuencia.

Son múltiples las cartas de edificación y biografías que escribe el P. Boroa, como la de los mártires del Caaró, la del mencionado D'Aragona, y los PP. Diego de Alfaro, Cristóbal de Mendoza, Juan Suárez y fundamentalmente la del P. Lorenzana. Pero incluso para el menologio de la Provincia Paraguaya, quien es el que lo inicia. Justamente un volumen entero, un tanto desorganizado o más bien inconcluso o fragmentado en partes, se encuentra en el Archivo romano de la Compañía de Jesús (ARSI), con el título "Paraq. 15. Necrolog. 1598-1702". Aunque en realidad abarca lo que en su interior se titula "Elogia Prov. Paraquariae" y justamente los tres primeros escritos hacen referencia a la vida de los PP. Barzana (1-I-1598), Añasco (12-IV-1605), D’Aragona (10-VI-1629) y Lorenzana (12-IX-1632), siendo firmados por el P. Boroa ${ }^{4}$,

bien della”, incluía personas que aún vivían y “es necesario que se dilate su impresión hasta que mueran”. El P. Justo Beguiristáin cree que la persona que aún vivía era el obispo Cárdenas y sus partidarios. El obispo falleció en 1668, diez años después que el P. Pastor y su manuscrito quedó archivado, aunque fue utilizado tanto por los PP. Del Techo como Lozano. Este último incluso lo cita, pero el original del P. Pastor se perdió (Baptista y Storni, 2001, III: 3065).

${ }^{4}$ ARSI Paraq. 15 necrológicas ff. 2-3v. 
y redactados para que sean leídas en la fecha recordatoria de su muerte. Es decir, escritos diferentes a las Cartas de Edificación, como escribe el mismo Boroa sobre D'Alagona, transcripta en este número de la revista. A estos "Elogios", le adjuntaron más adelante, en el libro mencionado, biografías más extensas, aunque sin fechas ni firma, como la del P. Ruiz de Montoya, a la que se suma una copia de su necrológica escrita en la Carta Anua del periodo 1626-1627. Luego sigue la autobiografía del P. Boroa que trataremos en particular, para continuar la lista con el P. Cnudder (apellido que se castellanizó como Crespo). Un nuevo folio abre otra sección del legajo, aunque con la misma tónica, con el título Elogia Patrum Societatis Jesu Provincia Paraquariae Sunt 15. Menologio Paraquariae y con las biografías de los PP. Barzana ( $† 1597)$, Torres (†1638), Boroa (†1657), Ransonnier (†1636), Cataldino (†1653), Mascetta $(\dagger 1658)$, Romero $(\dagger 1645)$, Osorio (†1639), Cavallero (†1711), Discreti $(\dagger 1669)$, Lorenzana (†1632), Altamirano $(\dagger 1704)$, Burges ( $\uparrow 1725)$, Solinas $(\dagger 1683)$ y Lizardi $(\uparrow 1735)^{5}$. A continuación la vida del P. Lorenzana, la más extensa, en estos momentos editada por Carlos A. Page. Finalmente se inscriben en latín unas amplias vidas de los PP. Romero, Márquez, Doménech, D'Otaro, Beizama, Ferrufino, Ricquart, Gallego Bonillo, Berthot, Serra y Donvidas ${ }^{6}$. Un folio final expresa que han sido extraídas del libro del P. Del Techo, aunque se puede decir que no todas, pues varias son de personas fallecidas después que el francés y otras explícitamente con la autoría del P. Boroa.

Otro menologio del Paraguay, o mejor dicho un catálogo de 14 mártires, se encuentra en Barcelona, como se señaló en otra oportunidad ${ }^{7}$, escrito posiblemente en 1715, que es la fecha de fallecimiento del último de ellos: Bartolomé de Blende, aunque no se incluye la del P. José Francisco de Arce, muerto tres meses después que aquel.

\section{La autobiografía}

En este contexto, es poco usual encontrarnos con este tipo de escritos autobiográficos $^{8}$. Al menos nosotros no los conocemos para la provincia del Paraguay. Podría decirse que la obra de Montoya es autobiográfica; pero no exactamente, pues aparece por una necesidad particular.

Al P. Boroa lo conocemos primeramente a través de las obras de los PP. Del Techo y Lozano, aunque su necrológica la escribió en la Carta Anua de 1658-16609 , el P. Simón de Ojeda ${ }^{10}$, quien fuera su compañero de viaje en 1610, dedicándole un obituario con el sustancioso título "Compendio de la vida, y actuación del venerable Padre Diego de Boroa, Provincial del Paraguay”, aunque bastante escueto en su contenido. Pero es la primera noticia biográfica de este misionero del que el P. Furlong anotó que una "Vida del P. Boroa se halla inédita en la Biblioteca Nacional de Madrid.

\footnotetext{
${ }^{5}$ Ibid, ff. $45-57 \mathrm{v}$.

${ }^{6}$ Ibid, ff. 234-340.

${ }^{7}$ Page, 2011: 22.

${ }^{8}$ San Ignacio escribió su autobiografía aunque en realidad se la dictó a su secretario, P. Gonçalves da Câmara, publicado 150 años después por los Bolandistas, con el título de "Relato del peregrino", hoy en: https://es.wikisource.org/wiki/Autobiograf\%C3\%ADa_de_San_Ignacio_de_Loyola

${ }^{9}$ Biblioteca del Colegio del Salvador, Carlos Leonhardt SJ (1927), Traducción de las Cartas Anuas del Paraguay 1658-1660, f. 78-79.

${ }^{10}$ El P. Simón nació en Montilla del Palancar, Cuenca, el 28 de octubre de 1589. Llegó a Buenos Aires en el referido grupo del P. Romero en 1610. Fue provincial de Chile (1643-1649), procurador en Europa por la provincia del Paraguay (1651-1658) y luego provincial (1658-1663). Falleció en Córdoba el 22 de setiembre de 1673 (Storni, 1980: 202).
} 
Nos. $18,577^{\prime 11}$. Pero lamentablemente no la hemos hallado con esa nomenclatura, ni en los catálogos de los manuscritos y ni siquiera alguien la menciona, como podrían haberlo hecho desde los PP. Del Techo, Jarque, Lozano o Charlevoix hasta Hernández o Astraín. Más curioso es que el mismo P. Furlong dejó dos trabajos inéditos para su colección de Escritores Coloniales Rioplatenses que tituló: "Diego de Boroa y su "Historia de los varones Ilustres", y "Diego de Boroa y su Elogio al P. Roque". Lo señaló Geoghegan, quien a su vez hace referencia de ellos ubicándolos en el "Archivo Guillermo Furlong SJ" del Colegio del Salvador ${ }^{12}$. Pero es del caso que nos informa el P. García Mata desde la institución, que tal archivo no existe y que desconocen el paradero de esta cuantiosa producción inédita.

Por lo que expresa al comienzo, el texto fue encargado por el provincial Diego de Torres, quien le solicita que: "apunte las misericordias que antes y despues que entro en la compañia”, como también "su vocacion a la misma compañía y a las yndias".

La autobiografía dedica la mayor parte de su espacio a la infancia e ingreso a la Orden, mencionando varios jesuitas de la provincia de Toledo, donde ingresó en 1605 y pareciera concluir luego de su viaje a Asunción y obviamente antes de concluido el mandato del P. Torres en 1615.

A sus padres no los menciona, pero sabemos que fueron Francisco López y Yolanda Álvarez de Boroa ${ }^{13}$, a quienes recuerda que lo habían criado:

"con mucho recojimiento y encerramiento porque eran temerosos de Dios, y anssi me castigavan severamente por qualquiera cossa en que faltasse. Murieron estando yo estudiando Latin en Trujillo de edad de trece años”.

Allí había nacido, el 25 de julio de $1585^{14}$, siendo después de aquella pérdida, cuando enfermó gravemente y un hermano suyo, que estudiaba Teología, lo fue a buscar "y me llevo consigo a Alcala para que estudie". En aquella tradicional ciudad con su prestigiosa universidad fundada por el cardenal Cisneros, conoció a los PP. de la Compañía de Jesús ${ }^{15}$ con quienes se confesaba, en tanto estudiaba Filosofía, aunque no lo motivaba ser religioso jesuita, mientras los jóvenes cistercienses, como su hermano, lo alentaban a sumarse a las huestes de San Bernardo. Al cuarto año de Filosofía tuvo contacto con el P. Nicolás de Almazán ${ }^{16}$, quien vino: "a hacernos platicas con mucha fuerca de espiritu y con ellas se comencaron a resucitar los desseos", sumándose sus frecuentes asistencias a la iglesia de San Francisco, a las capillas de Nuestra Señora del Carmen y del por entonces beato San Diego de Alcalá cuyo cuerpo se encontraba incorrupto en la Catedral. Toda esta mística le llevó a hacer una confesión general con

${ }^{11}$ Furlong, 1944: 492 (nota 172).

${ }^{12}$ Geoghegan, 1975: 493 y 494.

${ }^{13}$ ARSI, Paraq. 4.1 f. 1v. Catalogo Delos $P^{e s}$. y Hers ${ }^{o s}$. Que pasaron al Paraguay con el $P^{e}$. Ju ${ }^{a}$. Rom ${ }^{r o}$. Procurador Gnl. de aquella Provin ${ }^{a}$. Año de 1610.

${ }^{14}$ Storni, 1980: 42. Baptista y McNaspy, 2001: 499.

${ }^{15}$ Los jesuitas se asentaron en Alcalá de Henares muy tempranamente entre 1543 y 1546, siendo la primera residencia de la provincia de Toledo. Tenía el sentido que allí fue a estudiar Ignacio de Loyola antes que en París. Su primer superior fue el P. Francisco Villanueva (Alcázar, 1710, I: 15-16).

${ }^{16}$ Nicolás de Almazán (Valladolid, 1553-) era hijo de nobles, de los duques de Medinaceli, estudiando en Alcalá e ingresando a la Compañía de Jesús poco después. Fue designado ministro en el Colegio de Madrid y luego maestro de novicios en Villarejo. En 1607 fue designado provincial de Andalucía hasta que la Congregación General de 1608 lo desigó Asistente España, cargo que ocupó en dos oportunidades 1608 a 1615 y 1619 y 1631 (Alcazar, 1719, II: 283). 
el P. Francisco Rodríguez ${ }^{17}$ y comenzar a pensar en ingresar a la Orden de su hermano. Pero consultando con el sacerdote Julio Díaz Gutiérrez, devoto de la Compañía, le recomendó que hablara con el P. Almazán, quien definitivamente lo inclinó hacia la Orden de Ignacio.

Llegaba por entonces de Valencia a Alcalá, el famoso carmelita descalzo conocido como H. Francisco del Niño Jesús ${ }^{18}$, recibiendo aclamación del pueblo por su santidad "que le cortavan de sus vestidos". Diego se acercó y tuvo un intercambio de palabras sobre su ingreso como religioso que quedó resuelta, expresando luego que:

"Tratela con mi confessor, pareciole muy bien y con el Padre Almazán y Francisco Rodriguez y ambos me ofrecieron ayudarme en viniendo el Padre Provincial a Alcala (que siempre es por la quaresma) vino por muerte del Padre Luis de Guzman ${ }^{19}$ el Padre Julio García y los Padres le informaron con muchas muestras de voluntad”.

Habló con el provincial quien le preguntó si tenía ánimo para viajar a las Indias a lo que respondió que lo haría de buena gana, y el domingo de Ramos el provincial le comunicó a través del H. Martín de Padilla que fuera al colegio cuando quisiera, porque ya estaba admitido y a la mañana siguiente, juntó sus cosas y partió. Luego el provincial lo envió a la Casa de Probación de Madrid donde estaba el P. Luis de la Palma ${ }^{20}$ como maestro de novicios. Pero al poco tiempo el mismo P. Palma lo envió junto a otros novicios a Villarejo de Fuentes ${ }^{21}$ con el P. Juan Ponce de León ${ }^{22}$ para volver a entablar

${ }^{17}$ El P. Francisco Rodríguez fue recibido en el colegio de Alcalá de Henares de la Compañía de Jesús el 16 de marzo de 1567. Era natural de Aranda de Duero de la provincia de Burgos. Escribe su biógrafo que "era muy hábil y de buen talento en el púlpito, con espiritu y facilidad en que se exercitó algunos años: y, habiendo leído en Plasencia la Sagrada Theologia, fue enviado á Roma para predicar á los Españoles, y ser juntamente Secretario de la Asistencia de España en tiempos de el P. General Claudio Aquaviva. Despues de veinte años se volvió á la Provincia, en la qual travajo loablemente hasta su muerte" (Alcazar, 1710, II: 172).

${ }^{18}$ Su nombre era Francisco Pascual Sánchez (Villapalacios, 1544-Madrid, 1604), atendió el hospital de Antezana en Alcalá durante 27 años y luego ingresó a la Orden de los Descalzos. En Valencia fundó una "casa de mujeres arrepentidas" y atendió una peste que envolvió a la población. Regresó a Alcalá de paso hacia Madrid, donde fue efusivamente recibido como también señala su biógrafo, recalcando lo que manifiesta Boroa, que la gente le cortaba la ropa, "que fue necesario vestirle de nuevo" (Jesus Maria, 1624: 110).

${ }^{19}$ Era de Osorno y fue rector del colegio de Alcalá desde 1572 (Alcazar, II, 1710: 501).

${ }^{20}$ El P. Palma nació entre 1559 y 1560 en Toledo, España. Fue profesor de teología y filosofía, además de maestro de novicios y rector en varios colegios de la península, alcanzando a ser provincial de Toledo (1614-1617 y 1624-1627). Fue confesor y consejero de varios ministros de Felipe II, III y IV. Además que el general Vitelleschi le confió delicados asuntos de la Compañía de Jesús. Escribió varias reconocidas obras espirituales. Terminó sus días en el Colegio Imperial de Madrid, donde fue rector, falleciendo el 20 de abril de 1641 (Rodríguez Molero, 2001: 2960-2961).

${ }^{21}$ El noviciado de Villarejo de Fuentes, Cuenca, fue fundado por don Juan de Silva Pacheco y doña Gerónima de Mendoza al donar a la Compañía de Jesús los bienes necesarios para su edificación en 1501. El edificio contaba además con iglesia dedicada a San José (hoy iglesia de Santa María Magdalena) y escuela de niños. Perteneció a la provincia de Toledo y fue traslado a Madrid (Marín Barriguete, 2003: 519-558).

${ }^{22}$ El P. Juan Ponce de León fue recibido en la Compañía de Jesús de la provincia de Toledo el 22 de agosto de 1579 con 14 años, siendo natural de Marchena e hijo de don Luis Cristóbal Ponce de león, segundo duque de Arcos, marqués de Zahara, conde de Casares, señor del Estado de Marchena, Villagarcía y otros, y de doña María de Toledo y Figueroa, hija de los terceros condes de Feria, marqueses de Priego. Estuvo en los colegios de Murcia, Caravaca, Belmonte, Huete donde gobernó un trienio, Madrid y en la Casa de Villarejo donde fue ministro y ayudante de maestro de novicios, hasta que volviendo a ser rector de la misma falleció en ella el $1^{\circ}$ de febrero de 1606 (Alcázar, 1710, II: 582-583). 
el noviciado, cerrado por la pobreza que padecía aquel tiempo. Al llegar practicó los Ejercicios Espirituales de San Ignacio y se hizo muy devoto de la Virgen de Nuestra Señora del Popolo ${ }^{23}$ que se encontraba en un altar de la capilla. Inmediatamente relata varias acciones piadosas y señala que en la enfermería, se destacó más que en otro oficio y donde tuvo mucho trabajo.

Con respecto a su vocación por partir a las Indas, simplemente escribe: "Un dia del Beato Francisco Xavier me senti movido y desseoso de venir a las yndias a imitacion del Santo a quien tenia mucho amor y devoçion aun antes de entrar en la compañía”. Le contó esta inquietud al P. Palma quien le respondió que le parecía bien pero que "lo guardasse para su tiempo".

Posteriormente profesó los votos y fue enviado al seminario, donde siguió con devoción y admiración la vida de Francisco Javier, al momento que el viceprovincial P. Lucero $^{24}$ lo envió a Belmonte ${ }^{25}$ a enseñar latín. Antes de partir hizo los Ejercicios con el P. rector Bernardino Velasco ${ }^{26}$. Pasó por Villarejo y se encontró con el P. Palma y su ayudante. Repitió los Ejercicios, experiencia que no podríamos relatar como lo hace el propio P. Boroa, escribiendo:

"en el tiempo de las vacaciones me recoji diez dias a exercicios, los quales tuve de la passion particularmente en que nuestro Señor a el sea la gloria medio mas luz de aquellos sagrados misterios que nunca hasta entonces endereçando los muchos sentimientos que me dava en ellos a mi aprovechamiento y imitacionde sus sagradas virtudes y esto con mucho consuelo y devocion y desseos de mudança de vida y en estos exercicios me hiço nuestro Señor la soberana misericordia de llamarme a las indias pocos messes después segun mi quenta que va entro a fundar esta Provincia y fue de esta manera el $2^{\circ}$ dia de exercicios en la tarde que fue domingo en aquellas palabras qui vult venire post me ett medio nuestro Señor desseo de seguirle en afrentas, y desprecios, y de procurar cumplir la regla undecima y 12. Senti en el lavatorio de los pies mucho desseo de no luçir, y de officios humildes y entera sujeçion no solo a los superiores $e$

${ }^{23}$ Escriben Nieremberg y Andrade: “Ay en la Capilla del Noviciado de Villarejo de Fuentes vna Imagen deuotissima de nuestra Señora del Popolo, que por tradició se sabe que la traxo de Roma San Francisco de Borja, está rica, y curiosamente adornada, y es la Madre de aquel deuoto Nouidiado, Seminario de los lustroso y exemplar en Religion, y letras, que ha tenido esta Provincia (Andrade y Nieremberg, 1666: 640-641).

${ }^{24}$ El P. Hernando Lucero llevaba el mismo nombre que su padre, siendo natural de Alfaro, en La Rioja. Su madre era doña Juana de Melida, natural de Navarra. Su padre había sido criado del emperador Carlos V, sirviendo también a Felipe II. Hernando era el mayor de los hermanos, entrando en la Compañía de Jesús en 1570 a los 20 años. Se graduó como licenciado en Artes, siendo designado ministro del colegio de Alcalá, luego y a los 30 años fue rector del colegio de Plasencia y luego compañero de los provinciales Gil González Davila y Antonio Marcen. En 1589 fue designado rector del colegio de Alcalá por cinco años, luego visitador de la provincia de Andalucía y provincial de la de Toledo. Sucesivamente fue rector del colegio de Alcalá, viceprovincial, rector en Madrid y nuevamente provincial de Toledo, falleciendo en 1625(Alcázar, 1710, II: 284).

${ }^{25}$ Los jesuitas llegaron a Belmonte en 1558, estableciendo estudios de gramática. Su fundación quedó sin efecto y el ayuntamiento se hizo cargo con una limosna anual que se fue incrementando en el tiempo hasta que fue compartida en 1567 con el marqués de Villena y señor de Belmonte. Lo propio hicieron Juan de Zúñiga y su esposa, vecinos de Argamasilla de Alba en 1574. Casi una década después recibe otra importante donación y se crea un convictorio para alumnos externos que llegó a tener en 1569 cerca de 400 alumnos.

${ }^{26}$ El P. Velasco ingresó a la Compañía de Jesús el 9 de mayo de 1577 a los 16 años. Escribe Alcázar que unos le dan por patria a Madrid y otros a Burgos, criado por su tía doña Isabel de Osotio (Alcázar, 1710, II: 526).

124 Silvana M. Lovay. La autobiografía del P. Diego de Boroa... 118 a 129. 
iguales pero a los niños de la calle. Martes me dio nuestro Señor luz en mi propio conocimiento considerandome como un enfermo sin animo y que esto era de mio sin dios, mesmo sentimiento tuve a la tarde. La mediacion de ese dia era la oracion de el guerto y resignacion de Xristpo nuestro Señor en las manos de su Padre con que deje yo resignarme enteramente en sus manos aunque fuesse con cossa tocante a la mesma vida y en esta resignación me dio nuestro Señor desseo de venir a las yndias a dar la misma vida por su amor, ese mesmo dia con el mucho dolor de que Xristpo nuestro Señor derramasse sudor de sangre por mis pecados desee otra vez dar mi vida por su amor, y entiendo que $2^{a}$ vez venir a las indias”.

Estos inconmensurables deseos manifiestos en los Ejercicios por viajar a las Indias, lo decidieron contundentemente a hacerlo. Pasaron poco más de dos meses y medio, de larga oración y sentimientos, reflexionando sobre los posibles padecimientos que soportaría pero consolándose con la fuerza que Dios le daría. Dio cuenta de su vocación a "uno de los superiores, gran siervo de Dios y muy aficionado a los yndios", quien en oración constante, buscaron juntos la luz que definitivamente confirmara su vocación. No contentos con esto, escribieron al P. Palma solicitándole un consejo e incluso que lo tratase con el provincial Bartolomé Pérez ${ }^{27}$ que se encontraba en Alcalá. El rector hizo lo encomendado y el provincial le respondió: "Al Hermano Boroa puede $V R$ animar a esperar muchas misericordias de la mano del Señor y en señalle a que se disponga a ellas y a lo que desea". Por ese entonces pasaba el P. Alonso Mesía ${ }^{28}$, procurador del Perú, reclutando voluntarios para su provincia, como lo hacía también el de Japón. Pero el P. Boroa confiesa que no sintió que fueran aquellos sus destinos, sino la provincia del Paraguay, de donde también había arribado a Madrid su primer procurador el P. Juan Romero. El P. Palma intercedió con el sevillano cuando fue a Alcalá, con el ánimo de llevar gente al Paraguay. Pero sin aún tomar decisión, el P. Romero pasó a Roma, quedando la elección de los que lo acompañarían en el provincial P. Bartolomé Pérez. En tanto en Belmonte el P. Boroa recibía consejos del P. Jerónimo de Ribera, prefecto de espíritu del colegio, para que tuviera en cuenta en su viaje.

A su regreso de Roma, el P. Romero pasó por Belmonte y el P. Ribera lo consultó sobre si el provincial ya le había señalado sujetos, a lo que el procurador respondió que no, pero que llevaría con gusto al $\mathrm{H}$. Boroa por lo que le había dicho el $\mathrm{P}$. Palma. En tanto el provincial se trasladó a Villarejo, de donde envió mulas para buscar al rector del colegio y al $\mathrm{H}$. Boroa para entrevistarse personalmente y decidir si le permitía viajar. Salieron de Belmonte al anochecer, y al amanecer ya estaban en Villarejo cuando el provincial dudó en enviarlo, y determinó que lo decidieran los consultores. Alguna tensión e incertidumbre sufrió el P. Boroa quien habló con el compañero del provincial, el P. Robledillo, para ver qué podía hacer por él. Al tiempo que llegaban de Aragón para ir al Paraguay los PP. Juan de Humanes y el H. Francisco de Córdoba, con quienes compartió una misa el día de la Transfiguración ofrecida por el

\footnotetext{
${ }^{27}$ Bartolomé Pérez de Nueros (Calatayud, 1548-Alcalá de Henares, 1614) fue hijo del abogado fiscal del reino de Aragón Micer Joan Pérez de Nueros y doña Jerónima de Maynar, quien al fallecer fue enviado a estudiar a Belmonte con su tío. Ingresó a la Compañía de Jesús en Salamanca en 1564, donde estudió Filosofía y luego Teología en Valladolid y Salamanca. Fue profesor de Filosofía en Ávila y de Teología en Valladolid, además de profesor y rector del colegio de Alcalá. Fue designado provincial de Andalucía (1589-1594) y de Toledo (1608-1612), Asistente de España elegido por el P. Aquaviva en 1596. Fue uno de los mentores de la Ratio Studiorum y contribuyó a publicar las obras de los PP. Alfonso Salmerón y de Gabriel Vázquez (Medina, 2001: 723).

${ }^{28}$ En 1607 Alonso Mexia o Mesía fue electo procurador por la Provincia del Perú junto al P. Martín Funes a quienes se les otorgó 50 religiosos (Astraín, 1613, IV: 576).
} 
provincial. Al concluir, el P. Boroa fue llamado por el provincial y el maestro de novicios, quienes le comunicaron su decisión de aprobar su viaje, por lo que el joven expresa:

"me eche a sus pies con el agradecimiento que era raçon y nuestro Señor con la obediençia ya del Superior junto a mi desseo me dio luz de la gran conveniencia y singular providencia suya con que el Padre Provincial avia precedido en mi elección sintiendo mas altamente de mi vocacion provada en tantos contrastes y ultimamente de la obediencia”.

Continúa Boroa relatando que esa misma noche volvió a Belmonte para despedirse de compañeros y estudiantes. Uno de sus mejores amigos quiso ir con él y efectivamente lo hizo unos meses después, siendo su destino el Nuevo Reyno y su nombre Juan Gregorio ${ }^{29}$. Aguardó ocho días la llegada del novicio Simón de Ojeda, mientras la señora Da. Juana de Castilla les dio una "gruesa limosna de missas y oraciones”. Antes de partir el P. Boroa fue a Alcalá a despedirse del P. Palma y de su hermano

"por averme casi criado, y fue tan grande el sentimiento que tuvo de que le embiasse a deçir que se compussiesse con la compañía señalando al Padre Rector de Alcala en lo poco que tenia de legitima que pareciendole que avia gastado mas o, tanto en mis estudios y grados, no me quiso responder". Lo hizo luego con una extensa carta.

Partió el P. Boroa a Madrid a encontrarse con el P. Romero". Fue entonces que escribe: "Salimos de Madrid en un coche el Padre Martin Xavier de la Provincia de Castilla y los Padres Xristoval de la Torre y Juan de Alviz y los Hermanos Pedro Rodriguez y Simon de Oxeda y yo". Pasaron por Trujillo y Diego, luego de haber recibido una carta de su tía franciscana descalza, continuaron el viaje sin verla hasta llegar a Portugal donde hicieron los Ejercicios y tuvieron una confesión general de toda la vida ${ }^{30}$.

Ya embarcado, se encargó de cuidar las gallinas y los quehaceres del refectorio, no dejando más relato sobre el mismo. Al llegar a Buenos Aires ${ }^{31}$ fueron recibidos por el provincial a quien inmediatamente le expresó su deseo de ir a las misiones durante el camino a Córdoba. El superior atenuó su insistente pedido que fue creciendo en la medida que en el refectorio se leían cartas de los misioneros del Guayrá. Pero todavía no era tiempo de ello, y emprendió viaje a Santiago del Estero, donde tuvo contacto con los naturales, aprendiendo su lengua y recibiendo el trato afable que le brindaron, además de interiorizarse de las injusticias del servicio personal.

${ }^{29}$ El P. Juan Gregorio (Olite, Navarra, 1585 - Santafé de Bogotá, 1674), efectivamente hizo su noviciado en Villarejo, teniendo como rector al P. Luis de la Palma. Terminó su primer año de Teología y zarpó de Sevilla en la primera mitad de 1610 rumbo al Nuevo Reino de Granada, donde completó sus estudios y se dedicó a las misiones populares y fue el primer rector del colegio de Nueva Pamplona. Posteriormente llevó adelante los colegios de Tunja, Santafé, Mérida y de la universidad javeriana de Bogotá, donde falleció ciego (Méndez, 2001: 1812).

${ }^{30}$ Sus nombres los especifica Lozano (1755: 259), Pastells (1912: 175) y Astraín (1996: 46). De la provincia de Andalucía el P. Miguel de Sotomayor, el estudiante Cristóbal Diosdado y los coadjutores HH. Luis de Zayas, Francisco Naranjo y Diego de Sosa. De la provincia de Castilla los estudiantes Martín de Urtasun, Antonio de Ureña y Felipe Guevara. De la de provincia aragonesa los PP. Antonio Moranta, Juan de Humanes y Baltasar Seña con el coadjutor H. Diego Basaurigui. Finalmente de la provincia de Toledo los estudiantes Cristóbal de la Torre, Juan de Albiz, Simón de Ojeda y Diego de Boroa.

${ }^{31}$ Lo hicieron el 1 de mayo de 1610 (Storni, 1980: 42). Una relación de ese viaje en Page (2007: 51-54). 
Mientras el P. Torres se fue a Chile, el H. Boroa hizo los Ejercicios y se preparó para su sacerdocio ${ }^{32}$, ofreciendo su primera misa, mientras tenía su horizonte en las misiones de Asunción. Pero estaba dispuesto a ir donde le enviaran sin problema y hasta morir entre los indios. Así fue que lo enviaron a los calchaquíes con el P. Juan Darío, su superior. Pasó por Tucumán y de camino predicó a gran número de “indios y negros”. Viajaron detrás de las sierras a los diaguitas de Aconquija, Malle, Andalgalá y Guasan, donde aprendió el catecismo breve para poder hacer algunos sermones en su lengua y predicarles, logrando algunas conversiones, siempre acompañando al P. Dario, sobre todo en Andalgalá y varios sitios donde -según Lozano- levantaron 19 templos ${ }^{33}$. Regresó a Tucumán y de allí fue enviado a Córdoba y luego a Chile, donde atendió por un tiempo el hospital y le insistió al P. Torres para ir al Paraguay, quien finalmente accedió. Viajó a Mendoza con los novicios y el provincial, permaneciendo dos meses, de los que 20 días empleó en los Ejercicios. Expresando su gozo en un pasaje del escrito "sentí anssi mesmo mucho goço de verme ya soldado de Jesuxrispto sintiendo mucho consuelo y contento en ofrecer a servirle sin interés ni sueldo, y esto aunque el demonio me diera grandes premios".

Llegaron a Córdoba e hizo nuevamente los Ejercicios pues expresa "en orden a mi venida al Paraguay adonde tuve algun temor", sobre todo porque había escuchado que los guaycurúes estaban un tanto agitados. De Córdoba a Santiago del Estero y de allí a Asunción donde fue enviado a la misión de Guarambaré y el Itati con significativo agradecimiento $^{34}$.

Gratitud a su provincial "agradecido a quien tanto devo, aunque con el favor de Dios hare aun mas pero ofresco a VR los días que nuestro Señor me diere de vida, cada mes una missa, cada semana una diciplina, un rosario, la oracion y las demas buenas obras y exerçicios espirituales de aquel dia; y haçer especial memoria de VR como lo hago en la missa, oraciones, rossarios y en la letania. Siempre y ahora un mes de trabajo en aprender la lengua y el primer mes de catecismo, y yo todo por que todo me devo a vos con esto he satisfecho a lo que VR me mando sacado un tanto que de proposito le pondre en otro papel”.

Un gran futuro quedaba para este notable misionero que había escogido no solo la opción cristiana por los excluidos sino sobre todo ser uno más de ellos.

\section{Bibliografía}

Alcántara Bojorge, Dante A. (2009), "El proyecto historiográfico de Claudio Aquaviva y la construcción de la Historia de la Compañía de Jesús en la Nueva España a principios del siglo XVIII", Estudios de Historia Novohispana, nº 40, p. 69.

Alcazar, Bartholome (1719), Chrono-historia de la Compañía de Jesús en la provincia de Toledo y elogios de sus varones ilustres, fundadores, bienhechores, Fautores é Hijos Espirituales. Segunda parte, Madrid: Garcia Infançon impresor.

\footnotetext{
32 Obtuvo el sacerdocio el 15 de setiembre de 1610 de manos del obispo Trejo y Sanabria (Storni, 1980: 42).

${ }^{33}$ Page, 2010: 25-54.

${ }^{34}$ La región había sido señalada para su evangelización por el oidor Alfaro y los jesuitas escogidos fueron el italiano Vicente Griffi y el francés Baltasar Seña. El primero pasó a la orden franciscana (1621) y el segundo falleció prematuramente (1614), por lo que fueron sucedidos por los PP. Boroa y Juan de Salas (Del Techo, 2005: 254).
} 
Andrade, Alonso de y Juan Eusebio Nieremberg (1666), Varones Ilvstres en santidad, letras y zelo de las almas de la Compañia de Iesvs, T. V, Madrid: Ioseph Fernandez de Buendia.

Astraín SI, Antonio (1913), Historia de la Compañía de Jesús en la Asistencia de España, Tomo IV Aquaviva (segunda parte) 1581.1615, Madrid: Administración de Razón y Fe.

Astraín SJ, Antonio [1912-1929] (1999), Jesuitas, Guaraníes y Encomenderos. Asunción: CEPAG.

Baptista, J. y Mc Naspy, C. (2001), "Boroa (Beroa), Diego de. Misionero, superior”, en Charles E. O'Neill y Joaquín María Domínguez, Diccionario histórico de la Compañía de Jesús. Biográfico-Temático, T. I. Madrid: Universidad Pontificia de Comillas.

Baptista, J. y Storni, H. (2001), "Pastor, Juan. Misionero, superior, historiador", en Charles E. O'Neill y Joaquín María Domínguez, Diccionario histórico de la Compañía de Jesús. Biográfico-Temático, T. III. Madrid: Universidad Pontificia de Comillas.

Del Techo SI, Nicolás (2005) [1673], Historia de la Provincia del Paraguay de la Compañía de Jesús, Asunción: Centro de Estudios Paraguayos "Antonio Guasch"-FONDEC.

Del Techo SI, Nicolás y Orosz SI, Ladislao (1759), Decades vivorum illustium Paraquaiae Societatis Jesu. Ex Historia ejusdem Provinciae \& aliunde. Authore R.P. Nocilao del techo Ejusdem Societatis Gallo Belga Insulensi. Cum Synopsi Chronologica historiae Paraquariae. Parts Prima. Tyrnaviae: Typis Academicis Societatis Jesu.

Furlong SI. Guillermo (1944), Historia del Colegio del Salvador y de sus irradiaciones culturales y espirituales en la ciudad de Buenos Aires 1617-1943. T. I. Buenos Aires: Colegio del Salvador.

García Cárcel, Ricardo (2010), "Los jesuitas y la memoria histórica”, en Betrán Moya J. L. (ed.), La Compañía de Jesús y su proyección mediática en el mundo hispánico durante la Edad Moderna, Madrid; Silex.

Geoghegan, Abel Rodolfo (1975), Bibliografía de Guillermo Furlong SJ 1912-1974. Buenos Aires: Academia Nacional de la Historia.

Jesus Maria, P. Fray Ioseph de (1624), Historia de la vida y virtudes del venerable Hermano Fray Francisco del Niño Iesu. Religioso de la Orden de los Descalzos de N. S. del Carmen... Vcles; en el conuento de San Ioseph: por Domingo de la Iglesia.

Lozano, Pedro (1754/1755), Historia de la Compañía de Jesús de la Provincia del Paraguay, T. I y II, Madrid: Imprenta de la Viuda de Manuel Fernández.

Marín Barriguete, Fermín (2003), "La casa de probación de Villarejo de Fuentes en el siglo XVI: 'La madre de los novicios", en Javier Vergara Ciordia (coord.), Estudios sobre la Compañía de Jesús: los jesuitas y su influencia en la cultura moderna (s. XVI-XVIII), Madrid: UNED. 
Medina, F. B. (2001), "Pérez de Nueros y Maynar, Bartolomé. Teólogo, superior, asistente de España", en Charles E. O'Neill y Joaquín María Domínguez, Diccionario histórico de la Compañía de Jesús. Biográfico-Temático. Tomo III. Madrid: Universidad Pontificia de Comillas.

Méndez, D. (2001), "Gregorio, Juan. Misionero, predicador, superior”, en Charles E. O'Neill y Joaquín María Domínguez, Diccionario histórico de la Compañía de Jesús. Biográfico-Temático. Tomo III. Madrid: Universidad Pontificia de Comillas.

Page, Carlos A. (2007), Los viajes de Europa a Buenos Aires según las crónicas de los jesuitas de los siglos XVII y XVIII, Córdoba: Báez ediciones.

Page, Carlos A. (2010), "La evangelización jesuítica en el Valle Calchaquí. Hacia la idealización de un nuevo hábitat jesuítico-calchaquí", Revista Tempo da Ciência, Vol. 17. Núm. $33.1^{\circ}$ semestre.

Page, Carlos A. (2011), Siete ángeles. Jesuitas en las reducciones y colegios de la antigua provincia del Paraguay. Buenos Aires: sb ediciones.

Pastells SI, Pablo (1912), Historia de La Compañía de Jesús en la Provincia del Paraguay (Argentina, Paraguay, Uruguay, Perú, Bolivia, Brasil) según los documentos originales del Archivo General de Indias, T. I. Madrid: Librería General de Victorino Suárez.

Rodríguez Molero, F. (2001), "Palma, Luis de. Superior, escritor espiritual", en Charles E. O'Neill y Joaquín María Domínguez, Diccionario histórico de la Compañía de Jesús. Biográfico-Temático. Tomo III. Madrid: Universidad Pontificia de Comillas.

Storni SI, Hugo (1980), Catálogo de los jesuitas de la Provincia del Paraguay (Cuenca del Plata 1585-1768, Roma: Institutum Histopicum SI. 